\title{
Sulla caratterizzazione delle curve di diramazione dei piani tripli $\left({ }^{1}\right)$.
}

Nota di Ostak Chisini e di Caklo Felick lianaka (a Milano).

Snnto. - Si studiann e si caratterizzann, dal punto di vista aritmetico e funzionale, le curve di diramazione dei piani tripli. Nel presente lavoro la sudletta caratterizazione è limitata al caso dei piani tripli ottenuti per proiezion" di una superficie di ordine $\mathrm{n}(\mathrm{B})$. sprovvista di curva multipla, da un suo pumto multiplo secondo $\mathrm{n}-3$.

Si dimostra lunicitio birazionale del piano triplo avente ma data curva di dira. mazione e si di l'equazione effetliva di un suo modello proiettivo.

1. Introduzione. - $\grave{\mathrm{E}}$ noto che lo studio della curve di diramazione dei piani tripli pnò essere affrontato da un punto di vista strettamonte algebrico, profittando delle formule che dinno il discriminante dell'equarione cubica; ricerche in questo senso sono state fatte $\left(^{2}\right)$ tuttavia, pur essendosi ottenuti con questo mezzo notevoli risultati, non si può asscrire che essi escano dal campo della pura descrizione e classificazione dei piani tripli. Nel presente lavoro ci proponiamo di studiare alcuni problemi che non pare siano stati ancora posti oformulati in maniera precisa ed esplicita. Pensiamo che tali problemi, pertinenti alla caratterizazione delle curve di diramazione dei piani tripli, possano ridursi ai soguenti fondamentali :

A) Assegnare le condizioni caratteristiche (di tipo proiettivo e funzionale) sotto le quali una data curva $\varphi$ curva di diramazione di (almeno) un piano triplo;

B) Posto che una data curva $\varphi$ soddisfi alle condizioni suddette, determinare i piani tripli, birazionalmente distinti, che la posseggano come curva di diramazione.

Con riferimento ad un particolare modello proiettivo di piano triplo, quale si ottiono proiettando su di un piano una superficie $F$ di ordine $n$ (con $n \geq 3$ ) da un suo punto $O$ che sia $(n-3)-p l o$, noi ei limiteremo nel pre. sente lavoro a trattare quello che chiameremo il «caso semplice * di piano triplo, cioè il caso in cui la superficie $F$ non possegga curva multipla, nè propria nè infinitesima nell' intorno di $O$.

La trattazione di questo "caso semplice» çi permetterà di far vedere praticamente in qual modo intendiamo impostare e risolvere i problemi fon.

(i) Giunto alla Redazione nel gennaio 1946.

(2) Cfr. p. es.: G. Pomprat, Sulla rappresentazione algebrica dei piani tripli, "Rend. Sem. Mat. Univ. di Roma ", Serie $4^{a}, V_{o l}, 3^{\circ}, 1989$. Lo stesso A. ritornava sulla questione in: Osservazioni sui piani tripli "Rend. Ist. Jłomb. ", Vol. 74, 1940-41, studiando il compor. tamento dei piani tripli di fronte alle trasformazioni cremoniane. 
damentali che abbiamo sopra enunciati (cfr. i risultati contenuti nei teoremi dei $\S \S 3$ e 4 pag. 259 e 264) ed insieme di spianare la via alla trattazione di casi piǹ generali.

Prima di iniziare la trattazione vera e propria vogliamo qui ricordare una nota proposizione, dovuta all' EnRIQUes $\left({ }^{3}\right)$, secondo la quale se una certa curva $\varphi$ di ordine $m$, dotata di un certo numero di nodi e cuspidi, è di diramazione per un piano $n$-plo, lo stesso accade per ogni curva che si ottiene da $\varphi$ mediante una variazione continua che ne mantenga, insieme con l'or* dine, anche il numero dei nodi e delle cuspidi.

Le curve che in questa trattazione indicheremo con $\varphi$ saranno sempre curve di diramazione di piani tripli e quindi potranno evidentemente posse. dere soltanto cuspidi.

Nel seguito sempre riterremo che le curve di cui ei occuperemo siano curve generiche del sistena continuo a cui appartengono; restano quindi fin da ora eliminati tutti $i$ dubbi che potrebbero sorgere relativamente a speciali particolarizzazioni delle curve $\varphi$.

2. Curve covarianti, caratteri nnmerici e funzionali delle curve $\varphi$. Consideriamo dunque una superficie $F$ di ordine $n$ (con $n \geq .3$ ) avente un punto $(n-3)$-plo $O$ e realizzante il «caso semplice» di piano triplo, ciò sprovvista di curva multipla, propria o infinitesima nell' intorno di $O$. Supposto, come è sempre possibile, che $O$ sia il punto improprio dell'asse $z$, l'equazione di $F^{3}$ assumeria la forma

$$
F(x, y, z)=a_{n-3} z^{3}+3 b_{n-2} z^{2}+3 c_{n-1} z+d_{n}=0
$$

dove $a_{n-3}, b_{n-2}, c_{n-1}, d_{n}$ sono polinomii in $x, y$ di grado uguale al loro indice. Il nostro piano triplo potrá ritenersi disteso sul piano $x, y$ e la sua curva di diramazione $\varphi_{m}$, di ordine $m$, sarà allora definita come il luogo dei punti dello stesso piano per cui le soluzioni della (1) non sono tutte funzionalmente distinte, oppure (che è lo stesso) come traccia sul piano $x, y$ del cilindro, a generatrici parallele all'asse $z$, circoscritto alla snperficie $F^{\prime}$; l'equazione di $\varphi_{m}$ si otterrà pertanto uguagliando a zero il discriminante della (1)

(2) $\varphi_{m}=a_{n-3}^{2} d_{n}^{2}+4 a_{n-3} \theta_{n-1}^{3}-6 a_{n-3} b_{n-2} c_{n-1} d_{n}-3 b_{n-2}^{2} c_{n-1}^{2}+4 b_{n-2}^{3} d_{n}=0$.

Enunceremo qui alcune proprietà della $\varphi_{m}$ ehe, pur essendo o note $\left({ }^{4}\right)$ 'o di facile verifica, sono tuttavia da tener presenti perchè di grande importanza per la risoluzione dei nostri problemi fondamentali, risoluzione che affronteremo nei paragrafi seguenti. Interessano in primo luogo l'esistenza ed il

(3) F. ENRrores, Sulla costruzione delle funzioni algebriche di due variabili possedenti una data cuva di diramazione, "Annali di Matematiça s, Serie $4^{n}, 1,1924$.

(4) Cfr. per es.; i lavori di G. PoMreIs citati alla nota ( $\left.{ }^{2}\right)$. 
comportamento di alcune notevoli curve covarianti proiettive di $\varphi_{m}$. Si hanno anzitutto le tre curve seguenti

$$
\left\{\begin{array}{l}
\psi_{2 n-3}=\left|\begin{array}{ll}
a_{n-3} & b_{n-2} \\
c_{n-1} & d_{n}
\end{array}\right|=0 \\
\psi_{n n-2}=\left|\begin{array}{ll}
b_{n-2} & c_{n-1} \\
c_{n-1} & d_{n}
\end{array}\right|=0 \\
\psi_{2 n-4}=\left|\begin{array}{ll}
a_{n-3} & b_{n-2} \\
b_{n-2} & c_{n-1}
\end{array}\right|=0
\end{array}\right.
$$

che permettono di porre l'equazione della $\varphi_{m}$ nella forma seguente

$$
\varphi_{n}=\psi_{2 n-3}^{2}-4 \psi_{2 n-2} \psi_{3 n-1} \text {. }
$$

Si hanno poi le due curve

$$
\left\{\begin{array}{l}
\psi_{3 n-1}=a_{n-3-3} \psi_{2 n-3}-2 b_{n-2} \psi_{2 n-4} \\
\psi_{i n-3}=d_{n} \psi_{2 n-3}-2 c_{n-1} \psi_{2 n-2}
\end{array}\right.
$$

ognuna delle quali permette una seconda espressione dell'equazione della $\varphi_{m}$ come segue

$$
\left\{\begin{array}{l}
a_{n-3}^{2} \varphi_{m}=4 \psi_{2 n-4}^{3}+\psi_{3 n-6}^{2} \\
a_{n}^{2} \varphi_{m}=4 \psi_{2 n-2}^{3}+\psi_{3 n-3}^{2} .
\end{array}\right.
$$

In base a quanto siam venuti fin qui scrivendo sono di immediata dimo. strazione le proprieta seguenti :

a) le cuspidi di $\varphi_{m}$ sono date da quei punti di intersezione di $\psi_{2 n-3}$ con $\psi_{2 n-4}$ che non sono contemporaneamente intersezioni di $a_{n-3}$ e $b_{n-2}$; oppure da quei punti di intersezione di $\psi_{2 n-3}$ con $\psi_{2 n-2}$ che non sono contemporanea. mente intersezioni di $c_{n-1}$ con $d_{n}$. E di qui

b) tutte le nominate curve $\psi$ sono aggiunte della $\varphi_{m}$.

c) La $\psi_{2 n-1} \dot{e}$ tangente alla $\varphi_{m}$ ovunque la incontra fuori delle cuspidi, in un gruppo $T$ di $(n-2)(n-3)$ punti; la $\psi_{2 n-2}$ è pure tangente alla $\varphi_{m}$ ovunque la incontra fuori delle cuspidi, in un gruppo $T^{\prime}$ di $n(n-1)$ punti.

d) La $\psi_{2 n-8}$ seca su $\varphi_{m}$ fuori delle cuspidi il gruppo $T+T^{\prime}$, che è composto di punti distinti quando $a_{n-3}, b_{n-2}, c_{n-1}, d_{n}$ sono polinomii generici.

e) La $\psi_{3 n-6}$ è tangente in ognuna delle cuspidi di $\varphi_{m}$ alla relativa tangente cuspidale ed incontra $\varphi_{m}$ fuori delle cuspidi nei punti del gruppo $T$, avendo in ognuno di essi un nodo, con uno dei rami tangente a $\varphi_{m}$ e quindi possedendo ivi tre intersezioni riunite con $\varphi_{m}$ stessa. Analogo comportamento ha $\psi_{3 n-3}$ nelle caspidi e nei punti del gruppo $T$.

Un secondo gruppo di proprietà riguarda i caratteri aritmetici (o plückeriani che dir si voglia) della $\varphi_{m}$, caratteri che, tenuto presente quanto abbiamo detto alla fine del precedente paragrafo, si riducono a due fondamentali: 
l'ordine $m$ ed il numero delle cuspidi, numero che indichereno con $k$; essi son dati dalle formule

$$
\left\{\begin{array}{l}
m=4 n-6 \\
k=3(n-1)(n-2)
\end{array}\right.
$$

che sono di immediata dimostrazione.

Da quanto precede si deduce subito un terzo gruppo di proprietà della $\varphi_{m}$, proprietà queste ultime di tipo funzionale. Por formularle indichiamo con $K$ il gruppo delle cuspidi di $\varphi_{m}$ e con $R$ un generico gruppo di suoi punti allineati. Si ha allora

$$
\left\{\begin{array}{l}
T^{\prime} \equiv T+R \\
T+K \equiv(n-2) R
\end{array}\right.
$$

Si ha poi che la serie completa di tutti i gruppi equivalenti a $T+R$ ha dimensione non minore di 3 ossia

con $s \geq 0$.

$$
|T+R|=g_{n(n-1)}^{3+s}
$$

3. Caratterizzazione funzionale delle curve $\varphi$. -- Passiamo ora a risolvere i nostri problemi fondamentali, cioè a caratterizzare completamente (almeno nel «caso semplice» di cui ci occupiamo qui) le curve di dilamazione dei piani tripli e costruire questi ultimi a partire dalle curve stesse. Per quanto riguarda la caratterizzazione ricordiamo che essa dovrà necessariamente basarsi su altre proprietà delle curve oltre al possesso di dati caratteri plückeriani, poichè è noto che tale possesso $\dot{\theta}$ condizione necessaria ma non sufficiente perchè una data curva sia di diramazione per un piano multiplo $\left(^{5}\right)$; precisamente noi sfrutteremo le proprietà di tipo funzionale (8) ed $(8)^{\prime}$ che abbiamo enunciate alla fine del precedente paragrafo.

Consideriamo dunque una curva irriducibile $\varphi_{m}$ dotata di sole cuspidi $e$ possedente i caratteri plückeriani soddisfacenti alle relazioni aritmetiche proprie del «caso semplice» cioè un ordine $m$ tale che

$$
n=(m+6) / 4
$$

sia intero e un numero di cuspidi $k$ dato da

$$
k=3(n-1)(n-2) \text {. }
$$

Indichiamo ancora con $K$ il gruppo delle cuspidi di $e_{m}$ e con $R$ un generico gruppo di suoi punti allineati; sia poi $C$ un generico gruppo oanonico e $\psi_{i}$ una generica aggiunta di ordine $i$.

(5) Cfr. per es.: B. Segre, Sulla caratterizanzione delle curve di diramazione dei piani multipli generali, "Mem. Acc. d'Italia », Vol. I, n. 4, 1930. 
Sussiste il seguente

Teorema I. - La $\varphi_{\mathrm{m}}$ è curva di diramazione di un piano triplo se sono verificate le due seguenti ipotesi

1. - Il gruppo $\mathrm{K}$ è contenuto nella serie completa di tutti $i$ gruppi equi. valenti ai gruppi segati dalle curve di ordine $(\mathrm{n}-2)$ ossia esiste un gruppo $\mathrm{T}$ $d i+$ punti tale che $i l$ gruppo $\mathrm{T}+\mathrm{K}$ è equivalente a tutti $i$ gruppi segati dalle curve d'ordine $(\mathrm{n}-2)$; in formule

$$
T+K \equiv(n-2) R
$$

2. - La serie completa di tutti i gruppi equivalenti a $\mathrm{T}+\mathrm{R}$ non ha punti fissi e quindi ha dimensione non minore di 3 , ossia con $s \geq 0$.

$$
|T+R|=g_{n\{n-1\}}^{3+s}
$$

Più precisamente si ha che il piano triplo per cui tale $\varphi_{m}$ è di diramazione apparticne al «caso semplice».

La dimostrazione sarà ottenuta costruendo un piano triplo per cui la curva data è di diramazione, cioè dimostrando che nelle ipotesi poste esistono quattro polinomii in $x, y$ che indichiamo con $a_{n-3}, b_{n-2}, c_{n-1}, a_{n}$ tali che la $\varphi_{m}$ può scriversi come discriminante della equazione di terzo grado in $z$

$$
a_{n-3} z^{3}+3 b_{n-z} z^{2}+3 c_{n-1} z+d_{n}=0 \text {. }
$$

Avvertenza. - Prima di iniziare la dimostrazione avvertiamo che finora abbiamo usato indifferentemente una stessa lettera per indicare una curva algebrica ed un generico polinomio in $x, y$ che, uguagliato a zero, ne fornisce la equazione $\left({ }^{6}\right)$; d'ora innanzi quando ci occorrerà indicare un ben determinato tra questi polinomii useremo la lettera stessa (con la quale indichiamo la curva ed un polinomio generico) munita di una sopralineatura.

Premesso ció, passiamo alla dimostraziọne del teorema, dimostrazione che, per maggior chiarezza, daremo nol caso particolare in cui è $n=7$, avvertendo pero che la sua validità sussiste in generale, essendo il caso particolare trattato del tutto caratteristico.

Sia dunque una $\varphi_{m}$ per cui $n=7$ e quindi $n=22, k=90$; calcoli im. mediati dàno allora che il genere $p$ di $\varphi_{22}$ vale 120 e che il gruppo $T$ di cui l'ipotesi n. 1 afferma l'esistenza consta di $t=20$ punti; la relazione (9) allora diveati.

$(9)^{\prime}$

$$
T+K \equiv \mathbf{5} R
$$

Osserviamo anzitutto che la serie $|5 R|$ contiene la serie secata sulla $\varphi_{22}$ dalle quintiche e quindi è una $g_{110}^{20+s}$ con $s \geq 0$; essa risulta cosi speciale e verrà dunque secata dalla totaliıà delle curve aggiunte $\psi_{19}$ (d'ordine $19=22-3$ ) passanti per un residuo gruppo fisso di 128 punti, gruppo che indicheremo con $\Gamma$.

(5) 安 noto che tale polinomio è determinato soltanto a meno di una costante moltiplicativa. 
Possiamo dunque scrivere, indicando con $C$, come abbiamo già avvertito, un gruppo canonico,

$$
|5 R| \equiv|O-\Gamma|
$$

Inoltre non può una $\psi_{19}$ passare per i 110 punti comuni alla $\varphi_{22}$ e ad una quintica generica senza spezzarsi nella quintica stessa ed in una residua aggiunta $\psi_{14}$; possiamo dunque concludere che esiste una $\psi_{14}$ passante per il gruppo $\Gamma$.

Consideriamo ora la serie completa $|C+5 R|$ secata dalla totalità delle curve aggiunte $\psi_{24}$; ricordando le relazioni scritte qui sopra si ha:

$$
C+5 R \equiv \Gamma+10 R \equiv \Gamma+2 K+2 T
$$

esisterà dunque in base all'equivalenza qui scritta, una $\psi_{24}$ secante il gruppo $\Gamma+2 K+2 T$; tale $\psi_{24}$ dovrà avere quattro intersezioni riunite con $\varphi_{22}$ in ogni cuspide e quindi dovrà avere ivi un punto doppio, cioè sarà una biaggiunta a $\varphi_{22}$ secante fuori delle cuspidi il gruppo $I^{4}+2 T$. Ma abbiamo già visto che il gruppo $\Gamma$ è secato su $\varphi_{22}$ da una $\psi_{14}$; dunque. in base al classico teorema di NötHER, detto dell' $A f+B \varphi$, potremo scrivere

$$
\bar{\psi}_{24}=\bar{\varphi}_{22} \bar{f}_{2}+\bar{\psi}_{14} \psi_{10}
$$

e di qui deduciamo che il gruppo $2 T$ sta su una $\psi_{10}$ che è visibilmente una aggiunta. Come si verifica subito, tale $\psi_{10}$ è unica e non ha intersezioni con $\psi_{22}$ fuori dei punti $K$ e $T$.

Dal fatto che l'aggiunta $\psi_{10}$ seca su $\varphi_{22}$ (fuori dei punti doppi) il gruppo $2 T$, segue che la totalità delle $\psi_{10}$ secherà su $\varphi_{22}$ la serie completa di tutti $i$ gruppi equivalenti a $2 T+5 R$; ma per la relazione $(9)^{\prime}$

$$
2 T+5 R \equiv 2 T+K+T \equiv 3 T+K .
$$

Esisterà dunque una aggiunta $\psi_{15}$ secante il gruppo $3 T+K$; ossia tangent ${ }^{2}$ in ogni cuspide di $\varphi_{22}$ alla relativa tangente cuspidale ed avente tre intersezioni riunite in ognuno dei punti $T$. Facilmente si verifica che tale $\psi_{15}$ o unica e non ha in comune con $\varphi_{22}$ altro che i punti dei gruppi $K$ e $T$. Consideriamo ora la curva generica del fascio

$$
\psi_{15}^{2}+\lambda \psi_{10}^{3}=0
$$

essa ha l'ordine 30 e possiede essa pure una cuspide in ognuno dei punti $K$ (in eni $\psi_{10}$ e $\psi_{15}$ si intersecano semplicemente senza toccarsi) avendo ivi la stessa tangente cuspidale di $\varphi_{22}$. Essa ha quindi 6 intersezioni riunite con $\varphi_{22}$ in ognuno dei punti $K$ e, come si verifica facilmente, pure 6 in ognuno dei punti $\mathrm{T}$; in totale quindi $6.90+6.20=22.30$ intersezioni nei punti $K$ e $T$ e quindi nessuna altra intersezione variabile fuori di essi. Potremo allora imporre che una curva del fascio possegga una ulteriore intersezione con $\varphi_{22}$ ottenendo con cii una surva spezzata nella fog stessa ed in una residua $\vartheta_{8}$; 
con una scelta opportuna dei polinomii sopralineati rappresentativi delle curve in questione potremo allora scrivere

$$
\bar{\vartheta}_{8} \bar{\varphi}_{22}=4 \bar{\psi}_{10}^{3}+\bar{\psi}_{15}^{2} \text {. }
$$

Abbiamo fin qui ntilizzato la relazione (9)' che traduce l'ipotesi n. 1 del nostro teorema; fissiamo ora l'attenzione sull'ipotesi n. 2. Da essa si deduce immediatamente che esiste (almeno) una $\psi_{11}$ che contiene i punti $T$ ciascuno una sola volta $e$ quindi in particolare non è spezzata nella somma di una retta e della $\psi_{10}$. Infatti la totalita delle $\psi_{11}$ passanti semplicemente per i punti del gruppo $T$ seca la serie completa dei gruppi equivalenti a $T+R$, serie che, per ipotesi, non ha punti fissi.

Consideriamo ora la curva generica del fascio

$$
\varphi_{22}+\lambda \psi_{11}^{2}=0
$$

essa non ha intersezioni variabili con $\psi_{10}$, avendo con questa due intersezioni riunite in ognuno dei punti dei gruppi $K$ e $T$. Potremo allora imporre che una curva del fascio si spezzi nella somma della $\psi_{10}$ e di una $\psi_{12}$ residua; scegliendo opportunamente i polinomii sopralineati rappresentativi delle curve potremo sorivere

$$
\bar{\varphi}_{22}=\bar{\psi}_{11}^{2}-4 \bar{\psi}_{10} \bar{\psi}_{12}
$$

dove $\bar{\varphi}_{22}$ è lo stesso polinomio che compare nella (11) e $\bar{\psi}_{12}$ è visibilmente una aggiunta.

Osservando ora che la curva $\psi_{15}$ passa per $i$ punti comuni alla $\psi_{10}$ ed alla $\psi_{11}$, potremo applicare ad essa il teorema di NörHen già invocato sopra e scrivere

$$
\bar{\psi}_{15}=\bar{a}_{4} \bar{\psi}_{11}-2 \bar{b}_{5} \bar{\psi}_{10}
$$

dove $\bar{\psi}_{15}, \bar{\psi}_{10}$ e $\bar{\psi}_{11}$ sono gli stessi polinomii che compaiono nelle formule precedenti ed $\bar{a}_{4}=0, \bar{b}_{5}=0$ rappresentano una quartica ed una quintica. Per quanto riguarda la prima poi è facile convincersi che essa passa per i punti $T$. Infatti nella relazione (13) sopra scritta il primo membro rappresenta una curva che ha tre intersezioni riunite con $\varphi_{22}$ nei punti $T$; nel secondo membro il termine $2 b_{5} \bar{\psi}_{10}$ rappresenta una curva che ne ha almeno due; ne segue che la curva rappresentata dal termine $\bar{a}_{4} \dot{\psi}_{11}$ ne deve avere almeno altrettante; ma $\bar{\psi}_{11}$ ha intersezioni semplici con $\varphi_{22}$ nei punti $T$, dunque $a_{4}$ deve passare pure per essi.

Introduciamo ora nella (11) 1'espressione di $\varphi_{22}$ data dalla (12), tenendo conto della (13). Si ha

$$
\bar{\vartheta}_{8}\left[\ddot{\psi}_{11}^{2}-4 \bar{\psi}_{10} \bar{\psi}_{12}\right]=4 \bar{\psi}_{10}^{3}+\bar{a}_{4}^{2} \bar{\psi}_{11}^{2}-4 \bar{a}_{4} \bar{b}_{5} \bar{\psi}_{10} \bar{\psi}_{11}+4 \bar{b}_{5}^{2} \bar{\psi}_{10}^{2}
$$

ossia, raccogliendo i termini che contengono $\bar{\psi}_{11}^{2}$

$$
\bar{\psi}_{11}^{2}\left[\bar{\vartheta}_{8}-\bar{a}_{4}^{2}\right]=4 \bar{\psi}_{10}\left[\bar{\vartheta}_{8} \psi_{12}+\bar{\psi}_{10}^{2}-\vec{a}_{4} \bar{b}_{5} \bar{\psi}_{11}+\vec{b}_{6}^{2} \psi_{10}\right] .
$$


Ora il secondo membro di questa equazione è un poli romio divisibile per $\bar{\psi}_{10}$; tale deve dunque essere anche il primo membro. Ma abbiamo visto che $\psi_{11}$ non si spezza nella somma di $\psi_{10}$ e di una retta, mentre d'altra parte non può il polinomio di ottavo grado $\bar{q}_{8}-\bar{a}_{4}^{2}$ essere divisibile per $\bar{\psi}_{10}$ senza essere identicamente nullo. Si ha quindi identicamente

$$
\bar{\vartheta}_{s}=\bar{a}_{4}^{2} \text {. }
$$

La relazione (11) tenendo conto dell' identità ora dimostrata diviene

$$
\bar{a}_{4}^{2} \bar{\varphi}_{22}=4 \bar{\psi}_{10}^{3}+\bar{\psi}_{15}^{2} \text {. }
$$

Di qui si deduce che la quartica $a_{4}$ deve essere tangente a $\varphi_{22}$ nei punti $T$. Se infatti non lo fosse la curva complessiva $\bar{a}_{4 \varphi_{22}}^{2}$ avrebbe ivi una singolarità composta di un punto triplo con due rami tangenti tra loro ed il terzo no; il che si verifica facilmente essere impossibile in un punto che sia comune a $\psi_{10}$ ed a $\psi_{15}$ in quanto la sua equazione si scrive anche $4 \psi_{10}^{3}+\bar{\psi}_{15}^{2}=0$.

Ritorniamo ora sulla espressione (13); per quanto abliamo ora osservato, al secondo membro il termine $\vec{a}_{4} \bar{\psi}_{11}$ rappresenta una curva che ha tre intersezioni riunite con $\varphi_{22}$ in ognuno dei punti $T$; sappiamo che uguale proprietà ha la $\psi_{15}$. Dunque anche la curva $b_{5} \psi_{10}$ deve avere tre intersezioni riunite con $\varphi_{22}$ in ognuno dei punti $T$; ma abbiamo visto che $\psi_{10}$ ne ha due e non più, dunque anche la quintica $b_{5}$ dovrà passare per i punti $T$. Applichiamo allora il teorema di NöTher già invocato più di una volta alla curva $\psi_{11}$ che passa per i punti $T$, i quali esauriscono le intersezioni di $a_{4}$ e $b_{5}$. Avremo

$$
\bar{\psi}_{11}=\bar{a}_{4} \bar{\delta}_{7}-\bar{b}_{5} \bar{\gamma}_{6}
$$

dove $\bar{\gamma}_{6}=0$ e $\bar{o}_{7}=0$ rappresentano curve di ordine 6 e 7 rispettivamente. Analogamente possiamo fare per la $\psi_{10}$ la quale, essendo tangente a $\varphi_{: 2}$ e quindi alla $a_{4}$ in tutti i punti $T$ potrà scriversi

$$
\bar{\psi}_{10}=\bar{a}_{4} \bar{c}_{6}-\ddot{b}_{5}^{2}
$$

dove $\bar{c}_{6}=0$ rappresenta una curva di ordine 6 . Introduciamo ora queste espressioni nella (13); avremo, eseguiti i calcoli

$$
\ddot{\psi}_{15}=\bar{a}_{1}^{2} \delta_{7}-\bar{a}_{4} \bar{b}_{\overline{5}} \bar{\gamma}_{6}-2 \bar{u}_{4} \bar{b}_{5} \bar{c}_{6}+2 b_{5}^{3} .
$$

Se ora costruiamo l' espressione $4 \psi_{10}^{3}+\psi_{15}^{2}$ otteniamo

$$
\begin{gathered}
4 \bar{\psi}_{10}^{3}+\bar{\psi}_{15}^{2}=4\left(\bar{a}_{4}^{3} \bar{c}_{6}^{3}-3 \bar{a}_{4}^{2} \bar{b}_{5}^{2} c_{6}^{2}+3 \bar{a}_{4} \bar{b}_{5}^{4} c_{6}-\bar{b}_{5}^{6}\right)+ \\
+\bar{a}_{4}^{2}\left(\bar{a}_{4} \bar{\delta}_{7}-\bar{b}_{5} \bar{\gamma}_{6}-4 \bar{b}_{5} \bar{c}_{6}\right)^{2}+4 a_{4} \bar{b}_{5}^{3}\left(\bar{a}_{4} \bar{\delta}_{7}-\bar{b}_{5} \bar{\gamma}_{6}-2 \bar{b}_{5} \bar{c}_{6}\right)+4 \bar{b}_{5}^{6}= \\
=\bar{a}_{4}^{2}\left[4 \bar{a}_{4} \bar{c}_{6}^{3}-12 \bar{b}_{\overline{5}}^{2} \bar{c}_{6}^{2}+\left(\bar{a}_{4} \overline{\bar{c}}_{7}-\bar{b}_{5} \bar{\gamma}_{6}-2 \bar{b}_{5} \bar{c}_{6}\right)^{2}+4 \bar{b}_{5}^{3} \bar{\delta}_{7}\right]-4 \bar{a}_{4} \bar{b}_{5}^{4}\left(\bar{\gamma}_{6}-\bar{c}_{6}\right)
\end{gathered}
$$

e di qui, tenendo conto che, in base alla (11)' l'espressione sopra scritta deve essere divisibile per $\vec{a}_{4}^{2}$ otteniamo che o il polinomio $\bar{b}_{5}$ ha un fattore in comune con $\bar{a}_{4}$ o la differenza $\bar{\gamma}_{b}-\bar{c}_{b}$ è divisibile per $\bar{a}_{4}$. Ma, come si verifica 
subito dalle formule che dànno $\psi_{11}, \bar{\psi}_{10}$ e $\bar{\psi}_{15}$ la prima ipotesi è assurda giacchè porterebbe che $\psi_{15}$ e $\varphi_{22}$ avrebbero altri punti in comune oltre a quelli dei gruppi $K$ e $T$, contro la circostanza prima rilevata; sarà dunque

$$
\bar{\gamma}_{6}=\bar{c}_{6}+\bar{a}_{4} \bar{\sigma}_{2}
$$

dove $\bar{\sigma}_{2}$ rappresenta un polinomio di secondo grado.

Ritorniamo ora a considerare per un momento la formula (14); abbiamo dimostrato ohe è $\bar{\vartheta}_{8}=\bar{a}_{4}^{2}$, dal che consegue che il primo membro di tal formula è identicamente nullo. Lo stesso deve quindi avvenire anche del secondo membro; ma non potendo essere identicamente nullo il polinomio $\bar{\psi}_{10}$ sarà tale l'espressione tra parentesi, la quale, tenendo conto che è $\overline{\mathfrak{q}}_{8}=\bar{a}_{4}^{2}$, darà in definitiva

$$
\bar{a}_{4}^{2} \psi_{12}+\bar{\psi}_{10}^{2}-\bar{a}_{1} \bar{b}_{\overline{5}} \bar{\psi}_{11}+\bar{b}_{5}^{*} \psi_{10}=0 .
$$

Sostituendo in questa equazione le espressioni di $\psi_{11}$ e $\psi_{10}$ che son date dalle (16), (17) e tenendo conto della (18) otteniamo in definitiva

$$
\bar{\psi}_{12}=\bar{b}_{5}\left(\bar{\sigma}_{7}-\bar{b}_{5} \bar{\sigma}_{2}\right)-\vec{c}_{6}^{2}
$$

poniamo ora

$$
\delta_{i}-b_{5} \sigma_{2}=\bar{d}_{7}
$$

dove $\bar{d}_{\bar{t}}$ risulta essere, come $\bar{o}_{7}$, un polinomio di grado 7 ; otteniamo cosi

$$
\left\{\begin{array}{l}
\psi_{10}=\bar{a}_{1} \bar{c}_{6}-\bar{b}_{5}^{2} \\
\psi_{11}=\bar{a}_{4} \bar{d}_{7}-\bar{b}_{5} c_{6} \\
\psi_{12}=\bar{b}_{5} \bar{d}_{7}-\bar{c}_{6}^{2} .
\end{array}\right.
$$

Non resta ora che introdurre queste espressioni di $\psi_{10}, \psi_{11}, \psi_{12}$ nella (12) per ottenere

$$
\begin{aligned}
& \varphi_{22}=\left(\bar{a}_{4} \bar{d}_{7}-\bar{b}_{5} \bar{c}_{6}\right)^{2}-4\left(\bar{a}_{4} \bar{c}_{6}-\bar{b}_{5}^{2}\right)\left(\dot{b}_{5} \bar{d}_{7}-\bar{c}_{6}^{3}\right)= \\
& =\bar{a}_{4}^{2} \bar{d}_{7}^{2}+4 \bar{a}_{4} \bar{c}_{6}^{3}-6 \bar{a}_{4} \bar{b}_{5} \bar{c}_{6} \bar{d}_{7}-3 \bar{b}_{5}^{2} \bar{c}_{6}^{2}+4 \bar{b}_{5}^{3} \bar{d}_{7} .
\end{aligned}
$$

È così provato che la nostra $\bar{\varphi}_{22}$ può scriversi come il discriminante della equazione cubica in $z$

$$
\bar{a}_{4} z^{3}+3 \bar{b}_{5} z^{2}+3 \bar{c}_{6} z+\bar{d}_{7}=0
$$

cioè che essa è la curva di diramazione del piano triplo di equazione (1)'

4. Identità birazionale di due piani tripli possedenti la stessa curva di diramazione. - Il teorema testè dimostrato risolve in modo esauriente il problema fondamentale $A$ ) in quanto siamo ora in grado di affermare che $\theta$ certamente curva di diramazione di an piano triplo una $\varphi_{m}$ che possegga le 
proprieta caratteristiche enunciate al paragrafo precedente, cioè per cui sia

$$
\begin{gathered}
(m+6) / 4=n \quad \text { con } n \text { intero } \\
k=3(n-1)(n-2)=3\left(m^{2}-4\right) / 16 \\
T+T \equiv(n-2) R
\end{gathered}
$$

la serie $T+R$ sprovvista di punti fissi

e più precisamente il piano triplo di cui si tratta rientra nel «caso semplice».

Di più la tecnica dimostrativa seguita ci ha messi sulla via per una risposta esauriente anche del problema $B$ ), in quanto è stato costruito un piano triplo che ammette la curva data come curva di diramazione. Rimane ora a dirimere la questione se questo piano triplo sia unico (s'intende sempre nel campo birazionale) oppure ne esistano altri ; ed in questo secondo caso occorrerà costruirli. Noi risolveremo qui anche il problema fondamentale $B$ ) dimostrando il

TEorema II. - In relazione ad una curva $\varphi_{\mathrm{m}}$ soddisfacente alle condizioni sopra indicate esiste un unico piano triplo che la ammette come curva di diramazione.

Per semplicità e per fissare le idee conserveremo i simboli del precedente paragrafo e ragioneremo sul caso particolare ivi trattato; per dimostrare il nostro assunto ei baseremo sui risultati delle recenti ricerche del CHISINI relative alla identità birazionale di due piani multipli aventi la stessa curva di diramazione (7). Ivi è dimostrato che due funzioni algebriche di due variabili $z(x, y)$ e $\zeta(x, y)$ possedenti la stessa curva di diramazione $\varphi_{m}$ che sia dotata di soli nodi e cuspidi sono birazionalmente identiche sotto le due ipotesi seguenti :

1. - La curva $\varphi_{m}$ appartenga a quello che ivi è chiamato il «caso generale * cioè possa pensarsi ottenuta per variazione continua da una forma limite $\varphi_{m}^{\prime}$ spezzata in parti $C_{1}, C_{2} \ldots C_{n}$ tutte doppie, in modo che le intersezioni di due $C_{i}$ di indici consecutivi diano origine ciascuna a tre cuspidi di $\varphi_{m}$ e quelle di due $C_{i}$ di indici non consecutivi diano origine ciascuna a quattro nodi di $\varphi_{m}$.

2. - Le determinazioni di $z$ e $\zeta$ siano in numero maggiore di quattro.

Ora che la nostra $\varphi_{22}$ soddisfi all'ipotesi 1 . si trova già dimostrata al. trove ( $\left.{ }^{8}\right)$. Sembrerebbe invece che una funzione algebrica $z(x, y)$ definita da un piano triplo fosse in stridente contrasto con l'ipotesi 2., senonchè anche questa difficoltà è presto superata. Infatti nel lavoro citato del Cussisi tale ipotesi è necessaria per la validità generale di un lemma fondamentale (ivi

(i) O. Chlsins, Snlla identità birazionale di due funzioni algebriche di due variabili dotate di una medesima curva di divamazione, "Rend. Ist. Lomb. ", Vol. 77, Fasc. 20, 1943-44.

(*) O. Chisisr, Sulla cwrva di diramazione dei piani multipli, "Rent. Lincei ", Vol. 28. Serie $6^{\mathrm{n}}, 1^{\circ}$ sem. 1936. 
lemma 2) relativo ai sistemi di sostituzioni, lemma che afferma: "Due sistemi $S$ e $\Sigma$ ohe siano ugualmente concatenati e di cui l'uno $(S)$ sia transitivo su un numero $n \geq 4$ elementi, sono fra loro simili $"$.

Si presenta infatti, per $n \leq 4$ la eccezione fondamentale dei due sistemi: $S$ dato dagli scambii (12), (23), (31) e \ dato dagli scambii (12), (13), (14) che presentano la stessa concatenazione senza essere evidentemente simili. Ma si vede subito che, con l'ipotesi supplementare che i due sistemi in questione operino ambedue su tre elementi soltanto, il lemma sopra enunciato conserva in pieno la sua validita ; e questo è appunto il caso in cui ci si trova quando si tratta di dimostrare la identita birazionale di due piani che siano ambe. due tripli.

Sussistono dunque pienamente i risultati conseguiti dal Chrsins nel lavoro citato e rimane quindi dimostrata l'unicita del piano triplo costruito in relazione alla nostra $\varphi_{22}$. 